zurichten, juristische Gutachten nach allen Regeln der Zunft anzufertigen, scheint über alle Kritik erhaben.

Damit folgt die Kritische Justiz genau der unsinnigen Trennung von dogmatischen Fächern einerseits und Grundlagenseminaren andererseits, die zu einem der Probleme der universitären Ausbildung geworden ist. Auch die blumige Rhetorik von Studienordnungen kann nicht darüber hinwegtäuschen, daß die Lehrveranstaltungen, in deren Rahmen die Noten vergeben werden, die letztlich zählen, ab und an die Systematik, vor allem aber die Dogmatik spezifischer Rechtsgebiete vermitteln sollen, um den Studenten so in die Lage zu versetzen, einen Rechtsfall gutachterlich »ösen « zu können. Die hier erworbenen Kenntnisse sind durch Anfertigen mehrerer Gutachten unter Beweis zu stellen, ohne daß die Grundlagen dieser Arbeitstechnik und ihre verschwiegenen Voraussetzungen konkret an Fällen des spezifischen Rechtsgebiets Gegenstand der Ausbildung gewesen wären. Statt dessen findet die Beschäftigung mit diesen Grundlagen juristischen Handelns ausschließlich zu Beginn des Studiums statt, d. h. in einem Stadium, in dem die Studenten das materielle Recht noch nicht kennen. Juristische Methode wird also losgelöst von konkreten Anwendungssituationen und den hierbei überhaupt erst entstehenden Problemen vermittelt. Auf der Strecke bleiben damit sowohl die Probleme, denen die juristische Methode ihre Entstehung verdankt, als auch jene, die sie selbst verursacht. Die Ausbildungszeitschriften unterstützen diesen Trend mit ihren sogenannten "praktischen Fällen zum bürgerlichen-/Straf-/öffentlichen Recht«. Sie helfen, die Kunstfertigkeit im Anfertigen des »klausurstrategischen « Gutachtens einzuüben, sind damit Organ der herrschenden Rechtspraxis und letztlich der verlängerte Arm einer defizitären universitären Ausbildung. Eine Kritik ist von dieser Seite nicht zu erwarten. Sie wäre originäre Aufgabe der Kritischen Justiz.

\title{
Ninon Colneric Kritisches Rechtsdenken in der Praxis
}

In der schleswig-holsteinischen Arbeitsgerichtsbarkeit nahmen die Klageeingänge von 1990-I 997 um mehr als 50\% zu. Die Zahl der Richterstellen blieb unverändert. 1997 fielen bei den schleswig-holsteinischen Arbeitsgerichten infolgedessen pro Richter knapp 800 Verfahren an. Bei manchen Gerichten lag die Belastung noch deutlich über diesem Mittelwert. Es ist sehr schwer, unter solchen Rahmenbedingungen die Kraft aufzubringen, kritische Justiz zu praktizieren. In schätzungsweise $95 \%$ der Fälle entscheiden wir allerdings ohnehin alle gleich.

Mich persönlich beschäftigt nach wie vor die Frage, inwieweit unsere Rechtspraxis immer noch von Denkansätzen geprägt ist, die aus der Zeit des Nationalsozialismus stammen. Wir schleppen da einiges mit uns herum, dessen Ursprung wir ganz verdrängt haben. Ich denke beispielsweise an die gravierenden Umsetzungsdefizite, die es bei der Meinungsfreiheit im Arbeitsverhältnis gibt.

Oft hatte ich durch die Begegnung mit ausländischen Kolleginnen und Kollegen Schlüsselerlebnisse. Vor einiger Zeit sprach ich z. B. mit einem französischen Experten über den Schutz bei Kündigungen aùs unternehmensbezogenen Gründen. Ich übersetzte ihm die einschlägige Passage des deutschen Kündigungsschutzgesetzes und erläuterte ihm, wie die Gerichte sie interpretieren. Seine spontane Reaktion auf die Doktrin von der freien Unternehmerentscheidung: "Das ist ein Gesetzesbruch. Da steht doch `dringende betriebliche Erfordernisser. "Sein Entsetzen hat mir keine 
Ruhe gelassen. Ich habe für mich die ganze Geschichte des Schutzes gegen das, was wir in Deutschland "betriebsbedingte Kündigung «nennen, aufgearbeitet und bin dabei auf interessante Funde gestoßen. ${ }^{1}$ In der Weimarer Zeit wurde um die gerichtliche Überprüfbarkeit der unternehmerischen Entscheidung heftig gestritten. Unter den Nationalsozialisten setzte sich die Auffassung durch, daß weder die Notwendigkeit noch die Zweckmäßigkeit einer Maßnahme des Unternehmers in technischer, organisatorischer oder wirtschaftlicher Hinsicht nachzuprüfen sei. Die Literatur hatte dafür eine neue Rechtfertigung parat: das Führerprinzip.

Ich will Ihnen ein weiteres Beispiel nennen: Auf einer internationalen Tagung ging es darum, welchen Bindungen der Arbeitgeber im Rahmen des Arbeitsverhältnisses unterliegt, z. B. ob er die Arbeitnehmer gleichbehandeln muß. Soweit andere Systeme eine solche Gleichbehandlungspflicht bejahen, ist der Arbeitgeber der Bezugspunkt. Ich berichtete, daß die Gleichbehandlungspflicht des Arbeitgebers in Deutschland auf den Betrieb und nicht auf das Unternehmen bezogen wird. Eine italienische Studentin kommentierte dies mit der Bemerkung: "Das ist wohl immer noch euer Gemeinschaftsdenken.« Ich habe diese These später überprüft, und siehe da: Die Studentin hatte recht. Sie hatte sofort die ideologischen Wurzeln der Doktrin erkannt.

Ein ganz aktuelles Beispiel für erhellende Impulse, die die deutsche Arbeitsrechtspraxis aus dem Ausland erhält, ist eine Entscheidung zum Streikrecht, die das Arbeitsgericht Gelsenkirchen kürzlich gefällt hat. ${ }^{2}$ Sie setzt sich kritisch mit der in Deutschland herrschenden Doktrin auseinander, daß nur der gewerkschaftsgetragene Streik rechtmäßig sei und dieser wiederum nur tariflich regelbaren Zielen dienen dürfe. In den romanischen Ländern wird das Streikrecht demgegenüber als Individualrecht aufgefaßt. In einem großartigen obiter dictum äußerte das Gericht erhebliche Zweifel daran, daß das deutsche, durch Richterrecht geprägte Arbeitskampfrecht den Vorgaben des Art. 6 Nr. 4 der Europäischen Sozialcharta gerecht werde. Besonders bedenklich erscheine die mangelnde Anerkennung von spontanen, belegschaftsgetragenen Solidaritäts- oder Proteststreiks gegen unternehmerische Entscheidungen, deren Ziele sich nicht oder nur sehr bedingt in Tarifverträgen verwirklichen lassen. Es ist sicherlich kein Zufall, daß derartige Erkenntnisse gerade beim Arbeitsgericht Gelsenkirchen formuliert worden sind; denn dieses Gericht hat intensive Kontakte zu Frankreich. ${ }^{3}$

Eine der großen Herausforderungen für die Rechtsprechung ist zur Zeit der Arbeitnehmerbegriff, der wie das Arbeitskampfrecht im wesentlichen richterrechtlich geprägt ist. Es wäre zu wünschen, daß sich der Gesetzgeber des Themas annimmt. Er hat bessere empirische Voraussetzungen als die Gerichte, es in seiner ganzen Komplexität zu überschauen. Aber er hat bisher nicht die politische Kraft aufgebracht, es anzugehen. Notfalls müssen die Gerichte mit einer Neubestimmung des Arbeitnehmerbegriffs auf die grundlegenden Veränderungen des Erwerbslebens und der $\mathrm{Or}$ ganisation der Arbeit reagieren. Auch dazu hat es bereits eine eindrucksvolle Initiative aus der nordrhein-westfälischen Arbeitsgerichtsbarkeit gegeben. ${ }^{4}$

Der ideologische Druck, der gegenwärtig auf den Gerichten für Arbeitssachen lastet, ist enorm. Ich nenne nur die Stichworte Deregulierung, Globalisierung, Standort-

\footnotetext{
I Naheres dazu in einer Dokumentation des 5. Arbeitsrechtlichen Gesprachskreises der Otto Brenner Stiftung, der am 12.12.1997 in Frankfurt am Main zum Thema "Betriebsbedingte Kündigungen im Widerstreit « stattgefunden hat.

2 Urteil yom 13.3. I 998 - 3 Ca 3173/97-NZA-RR 1998, 352

3 Siehe z. B. Leo Kißler/Meinhard Zumfelde/Peter Jansen/Patrick Hunout: Arbeitskampfkulturen - Recht und Strategien von Streik und Aussperrung im deutsch-franzosischen Vergleich. - Frankfurt/New York 1997.

4 LAG Köln, Urteil vom 30.6.1995 - 4 Sa 63/95 - LAGE \$6r I BGB Arbeitnehmerbegriff Nr. 29.
} 
debatte. Man wirft dem Gesetzgeber und den Gerichten vor, durch überhöhte Schutzstandards zur Vernichtung von Arbeitsplätzen beizutragen.' Die Richterinnen und Richter werden z. B. durch die These, daß die Standards des deutschen Kündigungsschutzrechts im europäischen Vergleich besonders hoch seien, verunsichert. Ich habe dazu kürzlich ein Seminar an der Universität Bremen durchgeführt. Es zeigte sich: Die These ist unzutreffend. Es handelt sich um eine Mystifikation. ${ }^{6}$

$\mathrm{Ob}$ es, was die Rekrutierung der Richter betrifft, in der Arbeitsgerichtsbarkeit Besonderheiten gibt, die unter dem Blickwinkel kritischer Justiz von Interesse sein können, kann ich nicht generell sagen. In der schleswig-holsteinischen Arbeitsgerichtsbarkeit wird ein sehr hoher Maßstab angelegt. Die Proberichterstellen werden bundesweit ausgeschrieben. Nicht selten gibt es Bewerber mit einem »gut « in beiden Staatsexamina und einer besonderen arbeitsrechtlichen Vertiefung. Die könnten sich in der Wirtschaft eine goldene Nase verdienen, aber sie kommen trotz der sehr belastenden Arbeitsbedingungen und der vergleichsweise bescheidenen Vergütung zu uns. Ich glaube, daß wir mit dieser Methode einen guten Anteil kritischer Juristen »einfangen«.

Im Vergleich zu den anderen Gerichtszweigen ist die Arbeitsgerichtsbarkeit sicherlich nicht die Speerspitze der kritischen Justiz. Die Arbeitgeber sitzen ja mit am Richtertisch. Das ist eine Struktur, die ich immer verteidigen würde. Das Recht ist schwach, und seine Chancen, freiwillig angewandt zu werden, erhöhen sich auf diese Weise. Aber es ist keine Struktur, die die stringente Umsetzung kritischer Denkansätze besonders fördert. Aus der Habilitationsschrift, die Preis vor einigen Jahren vorgelegt hat, ${ }^{7}$ wissen wir z. B., daß die Arbeitsgerichtsbarkeit, verglichen mit der Zivilgerichtsbarkeit, bei der Vertragskontrolle in etlichen Punkten hinterherhinkt. Sie fragen: Was ist denn eigentlich »kritische Justiz «? Diese Fragen habe ich mir auch gestellt, und ich habe mich angesichts der Zusammensetzung des Plenums zusätzlich gefragt: Warum sind hier eigentlich so wenige Frauen ${ }^{8}$

Die Ausführungen im Forum I waren von einschüchternder Abstraktheit. ${ }^{9}$ Das war schon beim SDS so. Insoweit hat sich also nichts geändert! Ob das mitursächlich dafür ist, daß die Frauen hier so spärlich vertreten sind? »Boh, sind die schlau! Das kann ich nicht. Kann ich dann überhaupt etwas machen? « Ist das nicht vielleicht die Reaktion, die eine solche Sprache hervorruft?

Was den Maßstab kritischer Justiz betrifft, so lege ich für mein eigenes Handeln eine ganz schlichte Definition zugrunde: konsequente Durchsetzung der Grundrechte, Eindämmung willkürlichen Handelns Mächtiger.

In Großbritannien ist unter der Regierung Tony Blairs im Juli dieses Jahres ein Gesetz verabschiedet worden, das ganz auf dieser Linie liegt: der Public Interest Disclosure Act. Er dient dem Schutz der » whistleblowers«. Das sind Menschen, die auf Mißstände aufmerksam machen und dabei in einem Arbeitsverhältnis gewonnenes Wissen benutzen. Das britische Gesetz hat für die verschiedenen Fallgruppen ausgewogene Lösungen entwickelt. Ein solches Gesetz ist auch in Deutschland dringend erforderlich. Zwar könnten die Gerichte ähnliche Lösungen auf der Basis des Art. s GG auch hier entwickeln. Die Gerichte für Arbeitssachen haben damit aber allem Anschein

5 Exemplarisch Achim Schunder: Sicherung des Wirtschaftsstandorts Deutschland-Der Beitrag des Arbeitsrechts, NJW $1997,2654 \mathrm{ff}$

6 Siehe dazu auch den Bericht von Martine Le Friant über das dem Kündigungsschutz gewidmete XVI. Internationale Seminar der italienischen Vereinigung fur Arbeitsrecht und soziale Sicherheit vom 20. bis 25. Juli 1998 in ArbuR $1998,367 \mathrm{ff}$.

7 Grundfragen der Vertragsgestaltung im Arbeitsrecht, Neuwied/Kriftel/Berlin 1993.

8 Es hat sich auch auf der gesamten Veranstaltung keine einzige Frau aus dem Plenum zu Wort gemeldet, was ich noch auf keiner anderen juristischen Veranstaltung erlebt habe.

9 Die Autoren der Thesen haben diese nicht fur einen Abdruck freigegeben, die Red. 
nach infolge unserer unseligen Tradition größte Schwierigkeiten. Ich bekam kürzlich im Wege eines Wiederaufnahmeverfahrens den Fall einer Tierärztin auf den Tisch, die sich wegen eines nicht ordnungsgemäß aufgeklärten BSE-Verdachtes auf einem Schlachthof an die Öffentlichkeit gewandt hatte und deswegen fristlos entlassen worden war. Die Entlassung war in zwei Instanzen bestätigt worden. Die grundrechtliche Dimension des Falles ist in keinem der beiden Urteile auch nur angesprochen worden.

Bitte tragen Sie das Ihre dazu bei, daß auch in der Bundesrepublik ein whistleblowerGesetz verabschiedet wird. Es könnte uns wesentlich helfen, Menschen zu schützen, die anderswo in einer kritischen Praxis stehen.

\section{Reinhard Marx Das Asylrecht - kritisch gesehen}

Kritisches Rechtsdenken im Asylrecht hat zunächst nach einem rechtlichen Bezugssystem Ausschau zu halten. Die Verfassung hält lediglich den Begriff der politischen Verfolgung bereit. Den Gesetzgeber hat zwar seit Ende der siebziger Jahre eine enorme Regelungswut ergriffen. Die Regelungen betreffen jedoch nahezu ausschließlich verfahrensrechtliche Verschärfungen. Soweit materielle Rechtsfragen gesetzlich geregelt wurden, ging es ausschließlich um negative Abgrenzungskomplexe, wie etwa in Ansehung exilpolitischer Aktivitäten oder der materiellrechtlichen Bedeutung eines Voraufenthaltes in einem Drittstaat während der Flucht. Einen in sich schlüssigen und juristisch anwendungsfähigen Begriff der politischen Verfolgung hat der Gesetzgeber hingegen bislang nicht definiert und wird hiervon wohl auch für die absehbare Zukunft Abstand nehmen.

Das hierdurch entstandene Vakuum hat die Rechtsprechung des Bundesverwaltungsgerichtes ausgefüllt. Bis Ende der siebziger Jahre stellte das Gericht der Verwaltungspraxis geschmeidige Generalklauseln zur Verfügung, mit deren Hilfe mühelos innenund außenpolitischen Interessen Rechnung getragen werden konnte. Seit etwa seit Mitte der siebziger Jahre die Zahl der Flüchtlinge aus der Dritten Welt die der Ostblockflüchtlinge zu übersteigen begann, führte das Bundesverwaltungsgericht jedoch materielle Kriterien in das Asylrecht ein, um hierdurch den personellen Anwendungsbereich des Asylrechts einschneidend zu verengen. Inzwischen bezieht das Bundesverwaltungsgericht sogar mit drohendem Unterton gegen die menschenrechtsfreundliche Rechtsprechung des Europäischen Gerichtshofes für Menschenrechte Position.

Erst seit 1980 hat das Bundesverfassungsgericht seine Rechtsprechung zu materiellen Fragen des Asylrechts entwickelt. Die verfassungsgerichtliche Rechtsprechung zum materiellen Asylrecht ist zwar von einer gemäßigt liberalen Grundhaltung geprägt. Offen bleibt aber auch in der verfassungsgerichtlichen Rechtsprechung, welches Bezugssystem Basis des Asylrechts ist. So hat das Gericht sich zwar wiederholt auf die völkerrechtliche Regelungstradition des Asylrechts bezogen, errichtete dennoch zugleich einen schroffen Dualismus zwischen Verfassungsrecht und Völkerrecht. Vorherrschend ist deshalb bis heute eine scharfe Trennung zwischen dem verfassungsrechtlichen Asylrecht und dem völkerrechtlichen Flüchtlingsrecht. Seit I 994 ist das Bundesverwaltungsgericht sogar dazu übergegangen, anerkannte völkerrechtliche Grundsätze mit einem spezifisch deutschen Bedeutungsgehalt aufzuladen. 